\title{
Normal galaxies... in the infrared: AKARI Deep Field South data identifications and spectral energy distributions.
}

\author{
K. Malek* \\ Center for Theoretical Physics PAS, Poland \\ E-mail: malek@cft.edu.pl
}

\section{A. Pollo}

The Andrzej Soltan Institute for Nuclear Studies, Poland

Jagiellonian University, Poland

E-mail: apollo@ipj.gov.pl

\section{Shirahata, S. Matsuura}

Institute of Space and Astronautical Science (ISAS), Japan Aerospace Exploration Agency (JAXA), Japan

E-mail: sirahata@ir.isas.jaxa.jp, matsuura@ir.isas.jaxa.jp

\section{Kawada, T. T. Takeuchi}

Nagoya University, Japan

E-mail: kawada@u.phys.nagoya-u.ac.jp, takeuchi@iar.nagoya-u.ac.jp

\begin{abstract}
Far-infrared galaxy surveys provide us with a powerful tool to investigate, among others, the evolution of the star-formation history in the Universe, since they measure thermal emission from dust heated by the UV light from stars. We have carried out a deep survey AKARI Deep Field South (ADF-S) at 65, 90, 140 and $160 \mu \mathrm{m}$ with the FIS instrument on board the AKARI satellite. In order to minimize the contamination from the Galactic cirrus emission, we selected the region near the South Ecliptic Pole where the cirrus density is the lowest in the whole sky. The area of the ADF-S survey is $>12 \mathrm{deg}^{2}$. We have successfully detected almost 2200 sources down to $\sim 20 \mathrm{mJy}$ at $90 \mu \mathrm{m}$. Here we present the results of our search for counterparts of these far-infrared sources detected in the ADF-S in all available wavelengths in the public databases (NED, SIMBAD). For 500 sources brighter than $48.2 \mathrm{mJy}$ at $90 \mu \mathrm{m}$, we found counterparts for 330 objects, among them 314 known galaxies. We present the properties of these sources.
\end{abstract}

European Physical Society Europhysics Conference on High Energy Physics, EPS-HEP 2009,

July 16 - 222009

Krakow, Poland

\footnotetext{
* Speaker.
} 


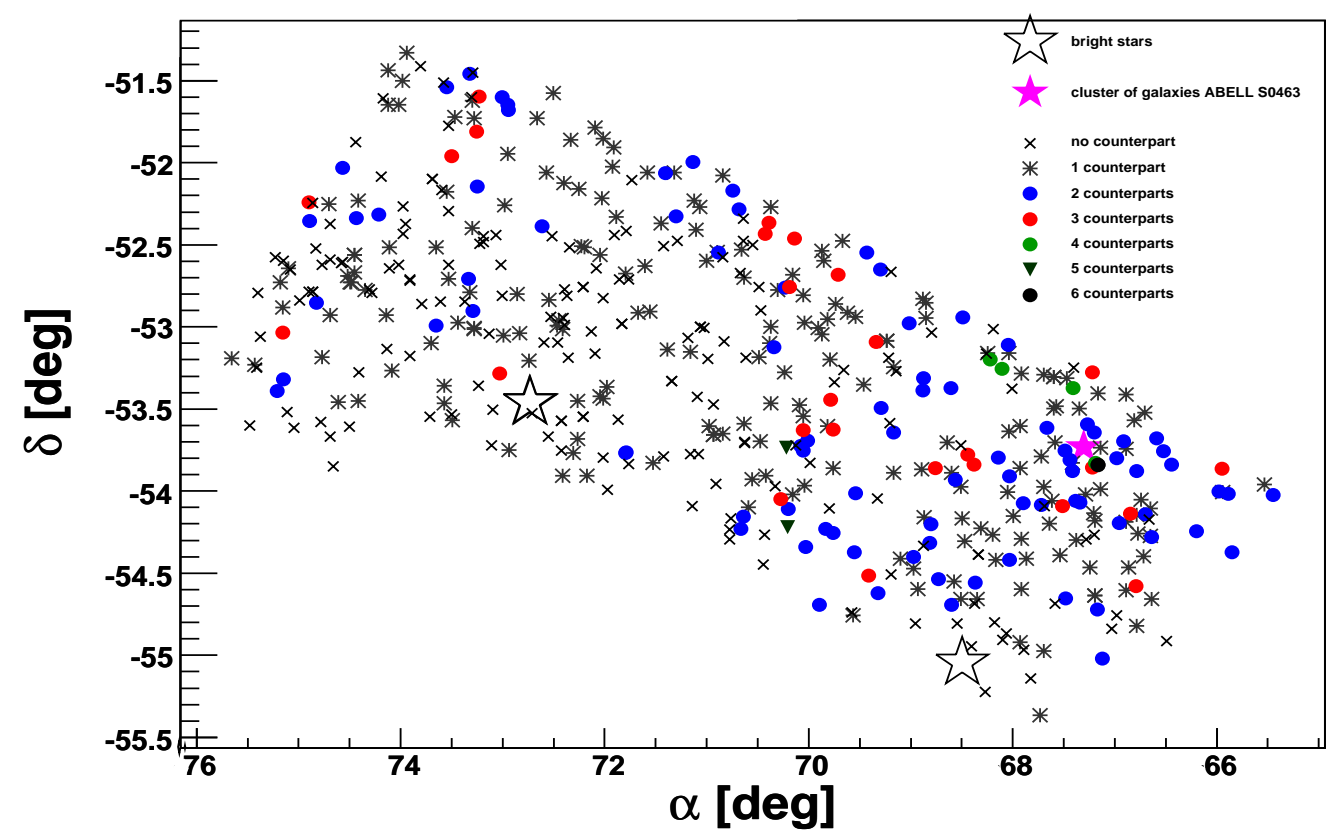

Figure 1: ADF-S field: positions of 500 sources brighter than $0.0482 \mu \mathrm{Jy}$ in the WIDE-S band (90 mum). Different colors indicate the number of counterparts found for each source in the radius of $40^{\prime \prime}$. Positions of two bright stars (big empty stars) present in the ADF-S area and the cluster of galaxies Abell S0463 at $z \sim$ 0.04 (big filled star) are also indicated. A large part of multiple counterparts is found in the region of the field corresponding to the cluster of galaxies.

\section{ADF-S sources: identifications and Spectral Energy Distributions (SEDs)}

We cross-correlated 500 ADF-S point sources brighter than 0.0482 Jy in the AKARI WIDE$\mathrm{S}(90 \mu \mathrm{m})$ band. We searched publicly available databases (NED and SIMBAD) for the ADF-S counterparts in all wavelengths in the radius of $40^{\prime \prime}$ on the sky, given the AKARI pixel size $\sim 26^{\prime \prime}$. In total, 500 counterparts for 330 ADF-S sources were found; 122 sources have more than one counterparts. We assume that the most nearby counterpart corresponds to the source, but we remain aware of the possible source confusion. The map of the identified sources in the ADF-S is shown in Figure 1. 314 of identified sources are known galaxies. Among them 173 galaxies were observed in the infrared before and 141 galaxies were not previously known as infrared sources. In addition, five stars, three infrared and six radio sources of an unknown origin and one quasar were found.

The far-infrared observations show us an otherwise hidden part of galaxies: the clouds of dust which normally cover birthplaces of stars. They allow us to investigate the dust properties and the properties of stars whose UV light is re-emitted by dust in the far infrared. Then, using four AKARI bands and other data, we constructed SEDs of our sources and modeled them to understand the composition and properties of dust and stars. As the simplest approach, we used modified black body fits to model the spectra of dust and stars. We applied also more sophisticated models for the dust emission in galaxies: [1] and [2]. An exemplary SED of the nearby dwarf starburst galaxy NGC 1705, hosting the biggest star cluster ever found (Super Star Cluster) is shown in Figure 2. 


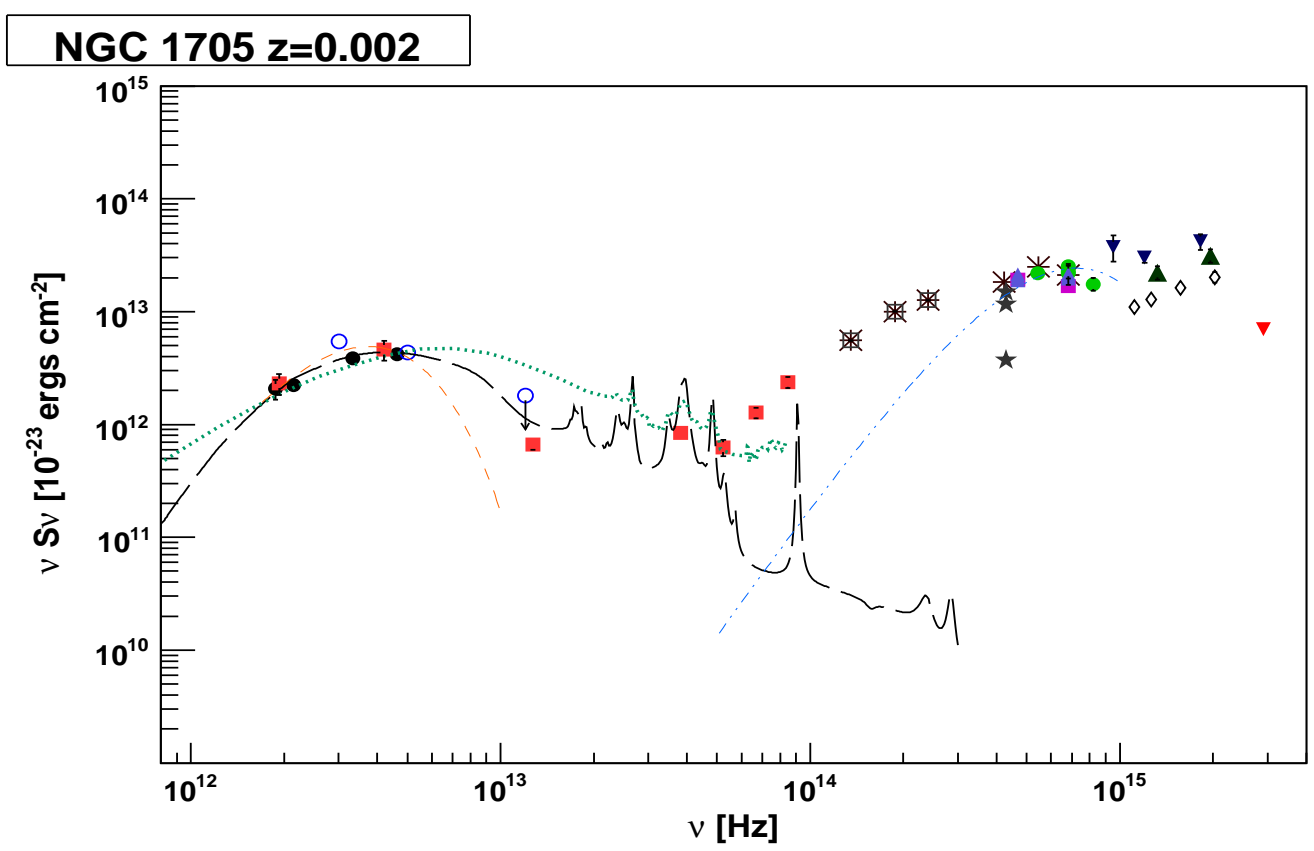

Figure 2: SED model of the nearby dwarf starburst galaxy NGC 1705.

\section{Conclusions}

The bright ADF-S sources are mostly nearby star-forming galaxies, containing a large amount of dust, but otherwise quite normal. However, many of them are classified as peculiar or belong to interacting systems of galaxies, which strengthens the evidence of the role of galaxy interactions in the stimulating the star forming processes in galaxies. AKARI observations allow for the first time the detailed observations of dust in these objects.

Acknowledgments This work is based on observations with AKARI, a JAXA project with the participation of ESA. We have made use of the NASA/IPAC Extragalactic Database (NED) operated by the Jet Propulsion Laboratory, Caltech, under contract with the NASA, and the SIMBAD database, operated at CDS, Strasbourg, France. We thank Misato Fukagawa for the information about the star candidates. This work has been supported (in part) by the Polish Astroparticle Physics Network. AP was financed by the research grant of the Polish Ministry of Science PBZ/MNiSW/07/2006/34A. TTT has been supported by Program for Improvement of Research Environment for Young Researchers from Special Coordination Funds for Promoting Science and Technology, the Grant-in-Aid for the Scientific Research Fund (20740105) commissioned by the Ministry of Education, Culture, Sports, Science and Technology (MEXT) of Japan and partially from the Grand-in-Aid for the Global COE Program "Quest for Fundamental Principles in the Universe: from Particles to the Solar System and the Cosmos" from the MEXT.

\section{References}

[1] D.A. Dale \& G. Helou, 2002, ApJ, 576, 159-168

[2] A. Li \& B.T. Draine, 2001, ApJ, 550, 213 